\section{(1) \\ CrossMark}

\title{
Coeliac disease and asthma association in children: the role of antibiotic consumption
}

\author{
Cristina Canova ${ }^{1}$, Gisella Pitter ${ }^{1}$, Jonas F. Ludvigsson ${ }^{2,3}$, Pierantonio Romor $^{4}$, \\ Loris Zanier $^{5}$, Renzo Zanotti ${ }^{1}$ and Lorenzo Simonato ${ }^{1}$ \\ Affiliations: 'Laboratory of Public Health and Population Studies, Department of Molecular Medicine, \\ University of Padua, Padua, Italy. ${ }^{2}$ Dept of Medical Epidemiology and Biostatistics, Karolinska Institutet, \\ Stockholm, Sweden. ${ }^{3}$ Dept of Pediatrics, Örebro University Hospital, Örebro, Sweden. ${ }^{4}$ Friuli-Venezia Giulia \\ Regional Health Information System, Informatica per il Sistema degli Enti Locali (INSIEL) S.p.A., Udine, Italy. \\ ${ }^{5}$ Epidemiological Service, Health Directorate Friuli-Venezia Giulia Region, Udine, Italy.
}

Correspondence: Cristina Canova, Laboratory of Public Health and Population Studies, Department of Molecular Medicine, University of Padua, via Loredan 18, 35131 Padua, Italy. E-mail: cristina.canovadunipd.it

ABSTRACT The relationship between coeliac disease and asthma has been scarcely investigated. Infant antibiotic exposure has been linked to both diseases. We evaluated the association between childhood coeliac disease and asthma and the role of antibiotics in the first year of life.

We followed a cohort of children born in 1995-2011 in the Friuli-Venezia Giulia region (Italy). Prescriptions for antibiotics in the first year of life and subsequent treated asthma were retrieved from drug prescription records; coeliac disease incident cases were identified from pathology reports, hospital discharges and exemption from prescription charges for clinical tests. We estimated incidence rate ratios (IRRs) using multivariate Poisson regression models.

Among the 143144 children, we identified 717 coeliac children and 34969 asthmatics. Children with asthma were at increased risk of coeliac disease (IRR 1.46, 95\% CI 1.25-1.67). Restricting the analysis to asthma that occurred before the diagnosis of coeliac disease, the excess risk disappeared, except for coeliac disease diagnosed after 5 years of age (IRR 1.37, 95\% CI 1.09-1.71). Antibiotics were not a confounding factor in these associations.

Childhood treated asthma and coeliac disease are significantly associated. This association is not confounded by antibiotic exposure in the first year of life and may be explained by other shared risk factors.

@ERSpublications

Coeliac disease associated with asthma in children is not explained by antibiotic exposure during the first year of life http://ow.ly/IJZ3Y

This article has supplementary material available from erj.ersjournals.com

Received: Oct 072014 | Accepted after revision: Jan 272015 | First published online: April 302015

Conflict of interest: None declared.

Support statement: This study was supported by a grant from the University of Padua to Cristina Canova (senior grant holder, year 2013, F.S. 4.18.01.05). Funding information for this article has been deposited with FundRef.

Copyright @ERS 2015 


\section{Introduction}

Coeliac disease and asthma are among the most common chronic diseases in children in the Western world [1]. Coeliac disease is a chronic immune-mediated small intestinal enteropathy triggered by gluten, which affects approximately $1 \%$ of the European population [2]. Villous atrophy (stage 3 according to the Marsh classification) detected on small-bowel biopsy is the gold standard for coeliac disease diagnosis [3]. Asthma is a chronic inflammatory airway disorder characterised by episodic airflow obstruction [4]. The diagnosis is challenging, especially in preschool children, due to lack of a single confirmatory test [4]. Although both diseases seem to have a genetic basis, environmental factors clearly play a major role in their aetiology.

To our knowledge, only three record linkage studies [5-7] have examined the possible relationship between coeliac disease and asthma. Although they all detected a significant positive association, they disagreed on its temporal direction (i.e. whether coeliac disease preceded asthma or vice versa).

Both surveillance bias and shared risk factors, which could be genetic, environmental or socio-medical, may explain the concurrence of the two disorders [6]. It is increasingly recognised that the commensal microflora plays an important role in the regulation of the immune system [8]. Antibiotic consumption during infancy, when the commensal microbiota is developing, may impair the microbiota-mediated mechanisms of immunological tolerance, thereby predisposing to inflammatory and allergic diseases [9]. Several epidemiological studies have found an association between antibiotic consumption in the first year of life and subsequent occurrence of asthma during childhood [10,11]. However, the same exposure has been scarcely investigated as a risk factor for coeliac disease $[12,13]$. In a large birth-cohort study based on record linkage of administrative data, we recently found a strong dose-dependent association between antibiotic exposure in the first 12 months of life and subsequent diagnosis of coeliac disease [14]. We therefore hypothesise that antibiotic consumption in the first months of life may explain the possible association between coeliac disease and asthma.

In the present study we investigated the association between coeliac disease and treated asthma in a large longitudinal registry-based population birth cohort followed for up to 17 years. Provided that an association was confirmed in our data, we aimed specifically at examining: 1) whether the association was bidirectional or one disease tended to precede the other; and 2) whether the association was confounded by antibiotic consumption during the first year of life (shared risk factor).

\section{Methods}

\section{Setting and study population}

The study population consisted of children born in the Friuli-Venezia Giulia (FVG) region in Italy between January 1, 1995 (first year of drug prescription availability) and December 31, 2011 (to allow $\geqslant 1$ year of exposure to antibiotics). FVG is covered by a regional integrated healthcare system developed in the 1980s with the goal of automatically collecting and pooling data on healthcare funded by the national health service using a unique regional identification code. This system and data sources available in the region have been described in detail previously [14].

The birth cohort was identified using the regional medical birth register (Certificato di Assistenza al Parto) which contains socio-demographic data on the parents, details of the pregnancy, labour and delivery, and the newborn at birth.

The following health data were used in this study (up to 2012): pathology reports from all the pathology departments in the region, coded using the Systematized Nomenclature of Medicine (SNOMED); hospital discharge records collected during episodes of inpatient care occurring within or outside the region with up to six diagnostic codes in the International Classification of Diseases, ninth revision (ICD-9); exemption from healthcare copayment coded in a national coding system; drug prescription records coded in the Anatomical Therapeutic Chemical (ATC) classification system; and mortality records.

\section{Definition of exposures and outcomes}

Antibiotic exposure was defined as the prescription of at least one antibiotic (ATC code J01*) during the first 12 months of life, identified from the drug prescription register.

Children were labelled as coeliac disease cases if they had at least one of the following [14]: a pathology report of villous atrophy (SNOMED codes D6218, D6318, M58, M58005, M58006 and M58007); a hospital discharge record with ICD-9 code 5790 in any diagnosis; an exemption from healthcare copayment with code I0060 according to the Italian national coding system. Concerning the latter data, all patients with coeliac disease can obtain clinical tests and gluten-free food free of charge from the national health service, provided they have a coeliac disease diagnosis with biopsy confirmation. The time of onset of coeliac disease was considered as the earliest date identifiable in the three sources of 
information (pathology, hospital and exemptions from copayment records). Incident coeliac disease cases were those children meeting the above-mentioned definition for the first time after 1 year of age.

In the main analysis, treated asthmatics (thereafter labelled as "asthma") were those who had at least two prescriptions, at different time points within a 12 -month period, of any of the following anti-asthmatic drugs: inhaled selective $\beta_{2}$-agonists (ATC code R03AC*); inhaled corticosteroids (ATC code R03BA ${ }^{\star}$ ); combined inhaled salbutamol/sodium cromoglycate (ATC code R03AK04); or combined inhaled selective $\beta_{2}$-agonists/corticosteroids (ATC codes R03AK06 and R03AK07). In a sensitivity analysis, prescription of inhaled selective $\beta_{2}$-agonists alone was not considered sufficient to define a subject as asthmatic. Concomitant use of inhaled $\beta_{2}$-agonists was allowed. A time interval of $<12$ months between the two prescriptions was required to better distinguish between asthmatics and children with sporadic consumption of anti-asthmatic drugs. By Italian law, each prescription is valid for 30 days and allows the dispensation of a maximum of two packages of the drug. The date of the earlier prescription of the two was considered the date of asthma onset. Incident asthma cases were those children meeting the above-mentioned definition for the first time after 1 year of age.

\section{Data analysis}

We explored the association between asthma and coeliac disease using Chi-squared statistics. In 69.3\% of children with both diseases, asthma diagnosis preceded coeliac disease diagnosis (see the results section for a more detailed explanation). Hence, in our main analysis we examined the effect of treated asthma (considered as exposure) on the risk of coeliac disease (considered as outcome). For this purpose, incident rate ratios (IRRs) and 95\% confidence intervals (95\% CI) were estimated using Poisson models, adjusted for year of birth and sex, according to the following steps: 1) we assessed the risk of coeliac disease associated with asthma occurring either before or after coeliac disease diagnosis; 2) we only considered asthma that preceded coeliac disease diagnosis, to avoid any chronological overlap between asthma and coeliac disease development; and 3) we included antibiotic consumption in the models.

Follow-up started at 1 year of age (i.e. from January 1, 1996 or later) and continued until the earliest of: the end of the study (December 31, 2012), death, migration out of the area or coeliac disease diagnosis. Children diagnosed with asthma or coeliac disease before the age of 1 year were not included in the analyses. Poisson models were stratified by age at coeliac disease diagnosis $(1-\leqslant 3$ years, $3-5$ years and $>5$ years), because early-onset coeliac disease may have different risk factors from late-onset coeliac disease. In this stratified analysis, coeliac disease subjects were compared with non-coeliac disease subjects having an equal or longer follow-up (i.e. $>2$ years or $>4$ years of follow-up for children with coeliac disease identified at 3-5 years of age or $>5$ years of age, respectively).

\section{Results}

The cohort consisted of 143144 children born in FVG between January 1, 1995 and December 31, 2011, corresponding to 1173692 person-years of follow-up. We identified 717 incident coeliac disease cases and 34969 incident treated asthma cases according to our main definition. In the sensitivity analysis with a more restrictive definition of treated asthma, we included 147199 children (1 197873 person-years of follow-up) and we identified 27899 incident asthma cases and 732 incident coeliac disease cases.

Our preliminary analysis (table 1) showed that asthma and coeliac disease coexisted in 254 children and were significantly associated $(\mathrm{p}<0.001)$. When we looked at the temporal relationship between the two disorders, we found that asthma diagnosis preceded coeliac disease diagnosis in 176 of the 254 cases $(69.3 \%)$ and followed coeliac disease diagnosis in the remaining 78 cases $(30.7 \%)$. Using the restrictive definition, asthma and coeliac disease coexisted in 214 children $(\mathrm{p}<0.001)$ (online table S1) and asthma preceded coeliac disease diagnosis in $142(66.4 \%)$ of the cases.

\begin{tabular}{|c|c|c|c|}
\hline & \multicolumn{2}{|c|}{ Coeliac disease } & \multirow[t]{2}{*}{ Total } \\
\hline & Absent & Present & \\
\hline \multicolumn{4}{|l|}{ Asthma } \\
\hline Absent & 107712 (99.57) & $463(0.43)$ & 108175 (100) \\
\hline Present & 34715 (99.27) & 254 (0.73) & $34969(100)$ \\
\hline Total & 142427 (99.50) & 717 (0.50) & $143144(100)$ \\
\hline
\end{tabular}

Data are presented as n (\%). p-value $<0.001$ using Pearson's Chi-squared test. 
We therefore examined the effect of treated asthma and early antibiotic consumption on coeliac disease onset (table 2). Incidence of coeliac disease was significantly increased in children with asthma, independently of the time of asthma diagnosis (IRR 1.46, 95\% CI 1.25-1.67). Antibiotic consumption during the first year of life was also significantly associated with subsequent coeliac disease (IRR 1.20, 95\% CI 1.03-1.39), but did not confound the relationship between asthma (that occurred before or after coeliac disease) and coeliac disease, since the effect of asthma on coeliac disease did not change more than marginally when we added antibiotics to the statistical model (adjusted IRR 1.44, 95\% CI 1.23-1.68). When the analysis was restricted to asthma occurring before coeliac disease diagnosis the risk disappeared (IRR 1.00, 95\% CI 0.84-1.20), again without any confounding effect of antibiotic treatment (IRR 1.00, 95\% CI 0.83-1.18). The sensitivity analysis using the restrictive definition of treated asthma led to similar results (online table S2).

Since early-onset coeliac disease may have different risk factors compared to late-onset coeliac disease, we stratified our analysis according to age at coeliac disease diagnosis (table 3): 194 (27\%) cases were diagnosed at age $1-\leqslant 3$ years, $178(25 \%)$ at $3-5$ years and $345(48 \%)$ at $>5$ years of age (mean \pm sD age $5.78 \pm 3.57$ years). With regard to asthma "exposure" occurring at any time (before or after coeliac disease diagnosis), the incidence of coeliac disease identified at $1-\leqslant 3$ years of age was increased in children with treated asthma (IRR 1.39, 95\% CI 1.02-1.89) and antibiotic consumption in the first year of life (IRR 1.56, 95\% CI 1.182.07). The incidence of coeliac disease identified at $>3$ years of age was increased in children with treated asthma, but not with antibiotic consumption. Antibiotic consumption during the first year of life was not a significant confounder for the relationship between asthma and coeliac disease stratified by age at diagnosis. When we excluded from the analyses 78 children with asthma occurring after diagnosis of coeliac disease, the incidence of coeliac disease identified at $1-\leqslant 3$ years of age was decreased in children with previous asthma (IRR $0.50,95 \%$ CI $0.31-0.78$ ). In contrast, the risk of coeliac disease identified at $>5$ years of age was significantly increased in children with previous asthma (IRR 1.37, 95\% CI 1.09-1.71) and was not confounded by antibiotic consumption during the first year of life (adjusted IRR 1.36, 95\% CI 1.09-1.70) (table 3). In the sensitivity analysis we obtained similar results (online table S3).

\section{Discussion}

We found a significant association between asthma (identified through prescriptions for anti-asthmatic drugs) and coeliac disease in a population-based birth cohort study of $>143000$ children. Incidence of coeliac disease was significantly increased among children with comorbid asthma identified at any time (i.e. either before or after diagnosis of coeliac disease) and this association was not explained by antibiotic consumption during the first year of life.

This is the first study that has examined the association between treated asthma and coeliac disease in children taking into account the role of early antibiotic exposure. Although enhanced medical surveillance may generate artificial clusters of disorders, the association between asthma and coeliac disease may also be explained by the presence of shared risk factors. The role of the commensal microflora for immune regulation

TABLE 2 Risk factors for coeliac disease for asthma occurring at any time and preceding coeliac disease, according to presence of asthma and antibiotic exposure

\begin{tabular}{|c|c|c|c|c|}
\hline & Coeliac disease & Person-years & Crude IRR $(95 \% \mathrm{CI})$ & Adjusted IRR $(95 \% \mathrm{CI})^{\#}$ \\
\hline Asthma (at any time) & 717 & 1173692 & & \\
\hline No & 463 & 832941 & 1 & 1 \\
\hline \multicolumn{5}{|l|}{ Any antibiotic in the first year of life } \\
\hline No & 408 & 707648 & 1 & 1 \\
\hline Yes & 309 & 466044 & $1.20(1.03-1.39)$ & $1.16(1.00-1-34)$ \\
\hline Yes & 176 & 340575 & $1.00(0.84-1.20)$ & $1.00(0.83-1.18)$ \\
\hline \multicolumn{5}{|l|}{ Any antibiotic in the first year of life } \\
\hline No & 372 & 707566 & 1 & 1 \\
\hline Yes & 267 & 465950 & $1.13(0.97-1.33)$ & $1.13(0.97-1.33)$ \\
\hline
\end{tabular}

Data are presented as $\mathrm{n}$, unless otherwise stated. Poisson regression models were adjusted for sex and year of birth. IRR: incident rate ratio.

\#: mutually adjusted for either asthma or antibiotic exposure; ๆ. excluding 78 asthma cases with a diagnosis of coeliac disease before diagnosis of asthma. 
TABLE 3 Risk factors for coeliac disease according to asthma and antibiotic exposure, stratified by age at coeliac disease identification for asthma occurring at any time and preceding coeliac disease

\begin{tabular}{|c|c|c|c|c|c|c|}
\hline & \multicolumn{2}{|c|}{$\begin{array}{l}\text { Coeliac disease identified at } \\
1-\leqslant 3 \text { years of age }\end{array}$} & \multicolumn{2}{|c|}{$\begin{array}{c}\text { Coeliac disease identified at } \\
3-5 \text { years of age }\end{array}$} & \multicolumn{2}{|c|}{$\begin{array}{c}\text { Coeliac disease identified at } \\
>5 \text { years of age }\end{array}$} \\
\hline & Crude IRR $(95 \% \mathrm{CI})$ & Adjusted IRR ${ }^{\#}(95 \% \mathrm{CI})$ & Crude IRR $(95 \% \mathrm{CI})$ & Adjusted IRR $(95 \% \mathrm{CI})$ & Crude IRR $(95 \%$ CI) & Adjusted IRR ${ }^{\#}(95 \% \mathrm{CI})$ \\
\hline Subjects & \multicolumn{2}{|r|}{194} & \multicolumn{2}{|c|}{178} & \multicolumn{2}{|c|}{345} \\
\hline Asthma (at any time) & $1.39(1.02-1.89)$ & $1.33(0.97-1.81)$ & $1.47(1.07-2.00)$ & $1.46(1.07-2.00)$ & $1.49(1.20-1.86)$ & $1.48(1.19-1.85)$ \\
\hline $\begin{array}{l}\text { Any antibiotic in the } \\
\text { first year of life }\end{array}$ & $1.56(1.18-2.07)$ & $1.52(1.15-2.02)$ & $1.05(0.78-1.42)$ & $1.02(0.75-1.38)$ & $1.09(0.89-1.36)$ & $1.06(0.85-1.31)$ \\
\hline Subjects & \multicolumn{2}{|r|}{155} & \multicolumn{2}{|c|}{150} & \multicolumn{2}{|c|}{334} \\
\hline $\begin{array}{l}\text { Asthma (before coeliac } \\
\text { disease diagnosis) }\end{array}$ & $0.50(0.31-0.78)$ & $0.47(0.30-0.75)$ & $0.80(0.54-1.17)$ & $0.80(0.54-1.18)$ & $1.37(1.09-1.71)$ & $1.36(1.09-1.70)$ \\
\hline $\begin{array}{l}\text { Any antibiotic in the } \\
\text { first year of life }\end{array}$ & $1.49(1.09-2.05)$ & $1.57(1.14-2.16)$ & $0.92(0.66-1.28)$ & $0.94(0.67-1.31)$ & $1.09(0.88-1.36)$ & $1.06(0.85-1.32)$ \\
\hline
\end{tabular}


and prevention of both inflammatory and immune-mediated disorders has received growing attention [8]. Moreover, early antibiotic consumption has been repeatedly associated with an increased risk of childhood asthma $[15,16]$ and, in a recent study, an increased risk of coeliac disease [14]. Therefore, we hypothesised that antibiotic consumption during the first year of life might act as a common risk factor for both asthma and coeliac disease by inducing an altered development of the infant's microbiota. Our results, while confirming the significant association between antibiotics and early-diagnosed coeliac disease that we previously reported [14], showed no confounding effect on the association between asthma and coeliac disease.

Other shared risk factors that we were unable to account for may explain the observed association between asthma and coeliac disease. For instance, vitamin D deficiency, which is common among coeliac $[17,18]$ and asthmatic patients [19,20], seems to have unfavourable effects on immune regulation [21], gut microbial balance and the intestinal mucosal barrier [22-24]. Therefore, vitamin D deficiency may be a common factor capable of increasing the risk of developing both coeliac disease and asthma. The possibility of a shared genetic basis should also be considered [25]. Moreover, as we based our definition of asthma on anti-asthmatic drug treatment, which is frequently prescribed to children for transient wheezing related to respiratory infections, we cannot discard the hypothesis that some infection related factor, rather than asthma, may lie at the basis of the association we describe herein. We also considered, in a sensitivity analysis, a restrictive definition which did not allow the prescription of inhaled selective $\beta_{2}$-agonists to be the unique criterion to define a subject as asthmatic. Inhaled corticosteroids, alone or in combination with inhaled $\beta_{2}$-agonists, and the association of inhaled salbutamol and sodium cromoglycate are mainly used as controller therapy for asthma [26]. As shown in the online supplementary material, analyses conducted with this restrictive definition confirmed the association between treated asthma and coeliac disease which was not explained by antibiotic consumption during the first year of life. To our knowledge, the relationship between coeliac disease and asthma has been evaluated by only three previous studies [5-7]. They all showed a significant positive association, but were not consistent with each other about its direction. In a record-linkage study of almost 60000 Finnish children, Kero et al. [5] found a seven-fold higher cumulative incidence of asthma at the age of 7 years in children with coeliac disease. However, the study did not establish whether coeliac disease occurrence preceded asthma or vice versa. A nationwide Swedish record linkage study [6] that examined the association between asthma and several autoimmune disorders exploiting hospital discharge data showed an increased risk of hospitalisation for coeliac disease occurring after the last hospitalisation for asthma (standardised incidence ratio 1.97, 95\% CI 1.64-2.34) in asthmatic subjects aged up to 20 years. Another Swedish record-linkage study of $>28000$ subjects with histologically diagnosed coeliac disease and 140000 matched controls [7] showed a 60\% increased risk of subsequent development of asthma in coeliac disease subjects, after adjustment for several covariates. Conversely, in the same study coeliac patients were more likely than controls to have a diagnosis of asthma preceding biopsy (OR 1.44, 95\% CI 1.34-1.56).

In the majority of the children included in this birth cohort, treated asthma preceded coeliac disease diagnosis. When the analysis was restricted to asthma that occurred before coeliac disease diagnosis, the incidence of coeliac disease among previous asthmatics was no longer increased. Stratification by age at coeliac disease diagnosis showed that subjects with previous asthma had a decreased risk of coeliac disease diagnosis at $1-\leqslant 3$ years, an unchanged risk at 3-5 years and an increased risk at $>5$ years of age; the sensitivity analysis confirmed these results. Such findings are probably due to the heterogeneous distribution among the three age-groups of the 78 (main analysis)/72 (sensitivity analysis) excluded cases with coeliac disease preceding asthma: most patients with coeliac disease identified at $1-\leqslant 3$ years of age developed asthma after coeliac disease diagnosis, while the majority of patients with coeliac disease identified at $>5$ years of age had already developed asthma before coeliac disease diagnosis. The observed chronological pattern may be related to the natural history of each disease and does not imply any causal relationship between them. Since diagnostic delay for coeliac disease can be as long as 5-10 years according to some reports $[27,28]$, we cannot exclude the possibility that coeliac disease onset preceded asthma in many cases.

Strengths of our study include the large population size, the longitudinal design with a long follow-up period and the availability of objective data, with no possibility of recall bias. The main limitation is the inability to directly verify the presence of asthma or coeliac disease, which is intrinsic in the use of administrative data. For asthma, in particular, since we had no data about diagnoses made by general practitioners or outpatient specialists, we used a surrogate indicator, anti-asthmatic drug treatment, which may have a low specificity for the disease, especially in early childhood. To minimise outcome misclassification, we required a minimum of two anti-asthmatic drug prescriptions within a 12 -month period to define a subject as asthmatic, based on the assumption that regular anti-asthmatic drug use helps to discriminate "true" asthma from transient wheezing. A similar definition was adopted in previous studies $[15,16]$. We decided not to include hospital discharge records in our asthma definition to avoid introducing a selection bias, considering that only a minority of asthma cases undergo hospitalisation during the course of the illness; furthermore, asthma, at 
variance with coeliac disease, has no defined diagnostic standard, therefore hospital diagnoses may be imprecise. In an attempt to increase the specificity of our definition we considered a more restrictive definition based on drugs mainly used as controller therapies for asthma. As expected, with this sensitivity definition the number of cases labelled as treated asthmatics roughly halved and was compatible with available figures of asthma morbidity in Italy [29]. In contrast, as regards coeliac disease, we are quite confident that our data truly reflect the presence of the disease, because 514 (72\%) of our 717 cases had a report of villous atrophy at small-bowel biopsy, which has been shown to accurately identify subjects with a clinical diagnosis of coeliac disease [30]. Another limitation is the possibility of surveillance bias, which may produce a spurious association between coeliac disease and asthma. Furthermore, due to the lack of specific data we could not examine the roles of dietary habits or genetics, which might explain the association between coeliac disease and asthma.

In conclusion, we found a significant association between coeliac disease and treated asthma in a large birth cohort of Italian children, but previous asthma only increased the risk of coeliac disease diagnosed after 5 years of age. Antibiotic exposure during the first year of life, a critical period in the development of microbiota and the immune system, increased the risk of early-diagnosed coeliac disease cases, but did not explain the concurrence of the two disorders in our data. Other shared genetic or environmental risk factors, as well as common biological pathways, may constitute the basis of this association, which deserves to be elucidated in further studies. Since a large percentage of infants are treated with antibiotics at some point, our findings have relevant public health implications, and, as any novel tested hypothesis, need to be further replicated.

\section{References}

1 Green PH, Jabri B. Coeliac disease. Lancet 2003; 362: 383-391.

2 Mustalahti K, Catassi C, Reunanen A, et al. The prevalence of celiac disease in Europe: results of a centralized, international mass screening project. Ann Med 2010; 42: 587-595.

3 Rubio-Tapia A, Hill ID, Kelly CP, et al. ACG clinical guidelines: diagnosis and management of celiac disease. Am $J$ Gastroenterol 2013; 108: 656-676.

4 Global Initiative for Asthma. Global Strategy for Asthma Management and Prevention. Revised 2014. www. ginasthma.org/local/uploads/files/GINA_Report_2014_Aug12.pdf

5 Kero J, Gissler M, Hemminki E, et al. Could TH1 and TH2 diseases coexist? Evaluation of asthma incidence in children with coeliac disease, type 1 diabetes, or rheumatoid arthritis: a register study. J Allergy Clin Immunol 2001; 108: 781-783.

6 Hemminki K, Li X, Sundquist J, et al. Subsequent autoimmune or related disease in asthma patients: clustering of diseases or medical care? Ann Epidemiol 2010; 20: 217-222.

7 Ludvigsson JF, Hemminki K, Wahlström J, et al. Celiac disease confers a 1.6-fold increased risk of asthma: a nationwide population-based cohort study. J Allergy Clin Immunol 2011; 127: 1071-1073.

8 Sommer F, Bäckhed F. The gut microbiota - masters of host development and physiology. Nat Rev Microbiol 2013; 11: 227-238.

9 Noverr MC, Huffnagle GB. The “microflora hypothesis” of allergic diseases. Clin Exp Allergy 2005; 35: 1511-1520.

10 Murk W, Risnes KR, Bracken MB. Prenatal or early-life exposure to antibiotics and risk of childhood asthma: a systematic review. Pediatrics 2011; 127: 1125-1138.

11 Penders J, Kummeling I, Thijs C. Infant antibiotic use and wheeze and asthma risk: a systematic review and meta-analysis. Eur Respir J 2011; 38: 295-302.

12 Myléus A, Hernell O, Gothefors L, et al. Early infections are associated with increased risk for celiac disease: an incident case-referent study. BMC Pediatr 2012; 12: 194-2431-12-194.

13 Mårild K, Ye W, Lebwohl B, et al. Antibiotic exposure and the development of coeliac disease: a nationwide case-control study. BMC Gastroenterol 2013; 13: 109-230X-13-109.

14 Canova C, Zabeo V, Pitter G, et al. Association of maternal education, early infections, and antibiotic use with celiac disease: a population-based birth cohort study in northeastern Italy. Am J Epidemiol 2014; 180: 76-85.

15 Kozyrskyj AL, Ernst P, Becker AB. Increased risk of childhood asthma from antibiotic use in early life. Chest 2007; 131: 1753-1759.

16 Marra F, Marra CA, Richardson K, et al. Antibiotic use in children is associated with increased risk of asthma. Pediatrics 2009; 123: 1003-1010.

17 Kemppainen T, Kröger H, Janatuinen E, et al. Osteoporosis in adult patients with celiac disease. Bone 1999; 24: 249-255.

18 Szymczak J, Bohdanowicz-Pawlak A, Waszczuk E, et al. Low bone mineral density in adult patients with coeliac disease. Endokrynol Pol 2012; 63: 270-276.

19 Gupta A, Bush A, Hawrylowicz C, et al. Vitamin D and asthma in children. Paediatr Respir Rev 2012; 13: 236-243.

20 Rajabbik MH, Lotfi T, Alkhaled L, et al. Association between low vitamin D levels and the diagnosis of asthma in children: a systematic review of cohort studies. Allergy Asthma Clin Immunol 2014; 10: 31-1492-10-31.

21 Dimeloe S, Nanzer A, Ryanna K, et al. Regulatory T cells, inflammation and the allergic response - the role of glucocorticoids and vitamin D. J Steroid Biochem Mol Biol 2010; 120: 86-95.

22 Cantorna MT, Munsick C, Bemiss C, et al. 1,25-Dihydroxycholecalciferol prevents and ameliorates symptoms of experimental murine inflammatory bowel disease. J Nutr 2000; 130: 2648-2652.

23 Liu W, Chen Y, Golan MA, et al. Intestinal epithelial vitamin D receptor signaling inhibits experimental colitis. $J$ Clin Invest 2013; 123: 3983-3996.

24 Ooi JH, Li Y, Rogers CJ, et al. Vitamin D regulates the gut microbiome and protects mice from dextran sodium sulfate-induced colitis. J Nutr 2013; 143: 1679-1686. 
25 Li Z, Ju Z, Frieri M. The T-cell immunoglobulin and mucin domain (Tim) gene family in asthma, allergy, and autoimmunity. Allergy Asthma Proc 2013; 34: e21-e26.

26 Papadopoulos NG, Arakawa H, Carlsen KH, et al. International consensus on (ICON) pediatric asthma. Allergy 2012; 67: 976-997.

27 Rampertab SD, Pooran N, Brar P, et al. Trends in the presentation of celiac disease. Am J Med 2006; 119: 355: e9-e14.

28 Norström F, Lindholm L, Sandström O, et al. Delay to celiac disease diagnosis and its implications for health-related quality of life. BMC Gastroenterol 2011; 11: 118-230X-11-118.

29 Galassi C, De Sario M, Biggeri A, et al. Changes in prevalence of asthma and allergies among children and adolescents in Italy: 1994-2002. Pediatrics 2006; 117: 34-42.

30 Ludvigsson JF, Brandt L, Montgomery SM, et al. Validation study of villous atrophy and small intestinal inflammation in Swedish biopsy registers. BMC Gastroenterol 2009; 9: 19-230X-9-19. 